\title{
Facial expression recognition and subthalamic nucleus stimulation
}

\author{
U Schroeder, A Kuehler, A Hennenlotter, B Haslinger, V M Tronnier, M Krause, R Pfister, \\ R Sprengelmeyer, K W Lange, A O Ceballos-Baumann
}

J Neurol Neurosurg Psychiatry 2004;75:648-650. doi: 10.1136/jnnp.2003.019794

Background: Deep brain stimulation (DBS) of the subthalamic nucleus (STN) improves motor signs in Parkinson's disease. However, clinical studies suggest that DBS of the STN may also affect cognitive and emotional functions. Objective: To study the impact of STN stimulation in Parkinson's disease on perception of facial expressions. Results: There was a selective reduction in recognition of angry faces, but not other expressions, during STN stimulation. Conclusions: The findings may have important implications for social adjustment in these patients.

$\mathrm{S}$ ubthalamic nucleus (STN) deep brain stimulation (DBS) has become an accepted form of treatment for patients with Parkinson's disease who have medically intractable motor symptoms. STN stimulation facilitates movement related frontal activity, ${ }^{12}$ but is not necessarily coupled with improvement in non-motor function. Clinical studies in patients with STN stimulation show subtle cognitive impairment. ${ }^{3-6}$ Furthermore, there is evidence for emotional changes, probably affecting social interaction-for example, STN stimulation can lead to poor behavioural control, ${ }^{5}$ mania, ${ }^{7}$ depression, anxiety, emotional lability, and social maladjustment. ${ }^{8}$

Facial expressions are powerful non-verbal displays of emotions, which signal valence information to others and are an important communication element in social interaction. Six basic emotions (happiness, surprise, fear, sadness, disgust, and anger) have distinct facial expressions and are expressed as well as perceived universally. ${ }^{?}$

Whereas previous studies of STN stimulation focused on emotional experience and behaviour, ${ }^{58}$ in the present study we investigated the effects of STN stimulation on emotion perception, using facial expressions. We hypothesised that STN stimulation has a negative influence on the perception of facial expressions of the emotions.

\section{METHODS}

\section{Subjects}

The study included six men and four women with idiopathic Parkinson's disease and bilateral DBS placed in the STN. Mean (SD) age was 61 (11.1) years, range 39 to 78, and the average duration of illness was 16 (3.1) years, range 12 to 20 . All except one of the patients were on levodopa (mean dose $600 \mathrm{mg} / \mathrm{d}$, range 0 to 1200), with entacapone in three patients. In addition, seven patients were treated with a dopamine agonists (bromocriptine, cabergoline, pergolide, pramipexole, or ropinirole).

Informed written consent according to the declaration of Helsinki was obtained from each subject. The local ethics committee of the Technische Universität München gave approval for the study.

\section{Neurosurgery}

The electrodes were implanted stereotactically in accordance with preoperative magnetic resonance and intraoperative stimulation. All patients received quadripolar electrodes (3387, Medtronic Inc, Minneapolis, Minnesota, USA) which had four platinum-iridium cylindrical surfaces $1.27 \mathrm{~mm}$ in diameter and $1.52 \mathrm{~mm}$ in length, with a $3 \mathrm{~mm}$ centre to centre separation. Contact 0 was the deepest, most distal contact. During the operation, the electrodes were adjusted to achieve the maximum beneficial effect on the motor symptoms. Brain magnetic resonance imaging (MRI) done a few days later confirmed the positions of the electrodes. The stimulation characteristics were as follows: mean pulse width 66 (12.7) $\mu$ s for all patients for both sides, mean frequency $143.5(22.6) \mathrm{Hz}$ for both sides, and mean voltage $2.91(0.78) \mathrm{V}$ for the right side and $2.69(0.65) \mathrm{V}$ for the left.

\section{Background neuropsychological assessment}

Neuropsychological assessments were done independently of the neurosurgical team, at least three months after implantation of the electrodes (mean 11 (7) months, range 3 to 24) with medication and stimulator ON for the whole day before testing recognition of facial expressions.

Background testing included the mini-mental state examination (MMSE).$^{10}$ To measure intelligence, we used either a German vocabulary test as an index of verbal intelligence $\left(\right.$ MWT-B $\left.{ }^{11}\right)$ or the German short version of the Wechsler adult intelligence scale, ${ }^{12}$ consisting of the subtests "information," "similarities," "picture completion," and "block design."

The Vistech vision contrast test system (VCTS) 6000 contrast sensitivity chart was used to test for basic visual processing. This test measures visual accuracy and contrast sensitivity. Higher visuoperceptual functions were assessed with the visual object and space perception test (VOSP), ${ }^{13}$ including the subtests "screening," "incomplete letters," "position discrimination," "number location," "dot counting," and "cube analysis." To establish that early processing stages of perception of faces were intact, the Benton recognition tes $\mathrm{t}^{14}$ was used. Scores of neuropsychological background tests are given in table 1 .

\section{Assessment of motor function and recognition of facial expressions}

After overnight withdrawal of symptomatic treatment, we measured the patients' motor functioning and ability to

\footnotetext{
Abbreviations: ACC, anterior cingulate cortex; MMSE, mini-mental state examination; UPDRS, unified Parkinson's disease rating scale; VOSP, visual object and space perception test
} 


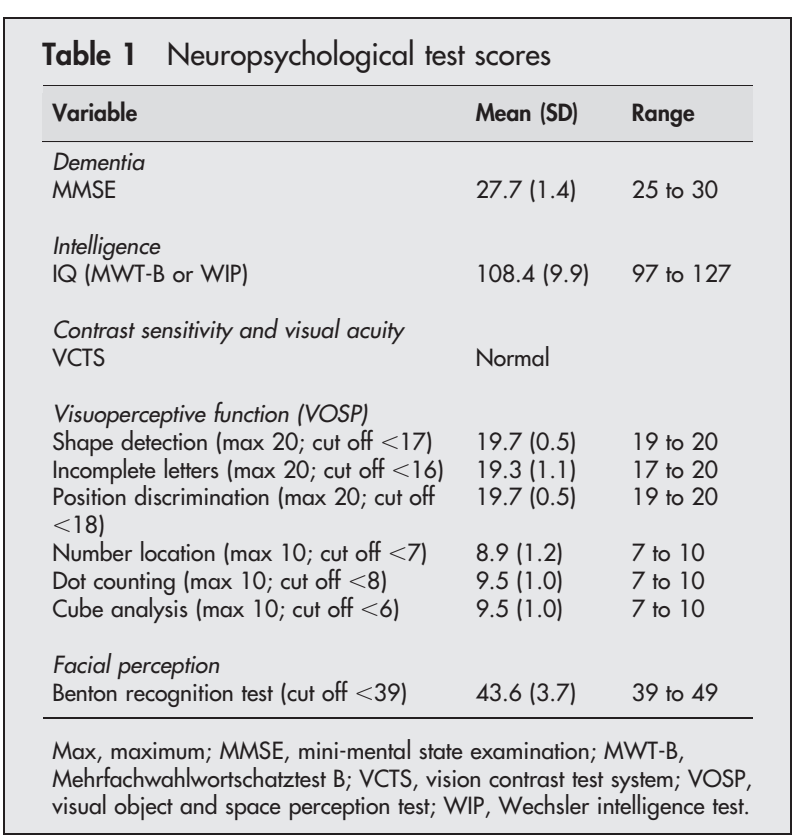

recognise facial expression with STN stimulation ON and OFF.

Motor symptoms ON and OFF stimulation were assessed with the motor score of the unified Parkinson's disease rating scale (UPDRS) ${ }^{15}$ and the Hoehn and Yahr rating, ${ }^{16}$ and subsequently compared using the non-parametric Wilcoxon test with one tailed $\alpha=0.05$.

To measure perception of facial expressions (happiness, fear, surprise, sadness, disgust, and anger) we used a set of computer transformed pictures showing faces of the actor JJ taken from the Facial expressions of emotions: stimuli and test (FEEST) ${ }^{17}$ Each face shows two emotions with different degrees of intensity (for example: 90\% happiness/10\% surprise; 70\% happiness $/ 30 \%$ surprise, $50 \%$ happiness $/ 50 \%$ surprise, $30 \%$ happiness/70\% surprise, and 10\% happiness/90\%surprise; for a detailed description, refer to the FEEST handbook ${ }^{17}$ ).

Patients were instructed to categorise each computer transformed face according to one of the six basic emotions. Participants gave verbal examples for the six basic emotions to assure their understanding of the meanings of the verbal emotion terms used for responses. Two sets of 30 pictures constituted one experimental block. Four experimental blocks were undertaken with the stimulator alternately switched ON and OFF between blocks. The initial setting of the stimulator (ON or OFF) was counterbalanced within the group. The maximum number of correct recognitions was 16 for each stimulator setting ( $\mathrm{ON}$ and $\mathrm{OFF}$ ) and each emotion (according to the test manual, ${ }^{17}$ ambiguous pictures with $50 \%$ of each emotion were excluded). Scores ON STN stimulation were compared with the OFF state using the non-parametric Wilcoxon tests with two tailed $\alpha=0.05$.

\section{RESULTS}

Significant improvement in motor function associated with stimulation ON compared with stimulation OFF was reflected in lower motor scores of the UPDRS (ON, 26.6 (12.6); OFF, $53.0(18.7) ; \mathrm{p}<0.05)$ and in the Hoehn and Yahr ratings (ON, $3.0(0.8)$; OFF, $3.6(0.8)$; $\mathrm{p}<0.05)$.

Recognition of angry expressions was selectively reduced during STN stimulation compared with the OFF state $(p<0.05)$. For all other facial expressions, the identification score did not differ between ON and OFF STN stimulation (cf. table 2).
Table 2 Average number of correct responses for each emotion during $\mathrm{ON}$ and OFF stimulation

\begin{tabular}{lrr}
\hline Emotion & \multicolumn{1}{l}{ ON } & \multicolumn{1}{l}{ OFF } \\
\hline Happiness & $15.9(0.3)$ & $15.6(0.7)$ \\
Surprise & $11.9(4.3)$ & $11.4(3.7)$ \\
Fear & $8.1(4.7)$ & $7.7(4.9)$ \\
Sadness & $11.5(5.0)$ & $11.9(5.0)$ \\
Disgust & $10.8(6.4)$ & $11.0(5.8)$ \\
Anger & $8.8(5.3)$ & $10.5(4.8)^{*}$ \\
\hline Values are mean (SD). & \\
${ }^{*} \mathrm{p}<0.05$ & \\
\end{tabular}

\section{DISCUSSION}

In this study we investigated perception of emotional expressions in Parkinson's disease patients with STN stimulation. STN stimulation selectively reduced the recognition of angry faces, while recognition of all other emotional expressions was unaltered. Although our study population is small, the selective reduction in recognition of angry facial expressions during STN stimulation was remarkable and may contribute to the understanding of behavioural dyscontrol and social maladjustment previously reported in STN stimulated patients with Parkinson's disease. ${ }^{5}$

In a previous neuropsychological study, perception of anger and disgust was reduced in unmedicated Parkinson's disease patients. ${ }^{18}$ While these results seem to resemble our results in unmedicated patients, there is an important difference-in our study reduced anger perception was independent of drug treatment (patients were off drug treatment throughout the experiment) and can only be attributed to STN stimulation.

The human STN is a small nucleus with average dimensions of $3 \times 8 \times 12 \mathrm{~mm}$ (coronal/sagittal/axial), which is densely surrounded by fibre tracts. Animal studies suggest that the STN can be divided into a limbic (medial), a sensorimotor (dorsolateral), and a cognitive-associative (ventromedial) part (for a review, see Parent and Hazrati ${ }^{19}$ ). Owing to current spread occurring during DBS, an electrode centred in the sensorimotor part of the STN may co-stimulate the limbic STN. The medial tip of the STN in animals is principally targeted by limbic cortices ${ }^{19}$ such as the orbitofrontal cortex and the anterior cingulate cortex (ACC). ${ }^{20} \mathrm{~A}$ close functional connection between the STN and the ACC has been further underlined by a positron emission tomography study in Parkinson's disease with STN stimulation. ${ }^{6}$ Moreover, the ACC has been associated with the perception of angry faces in a functional imaging study. ${ }^{21}$ We therefore propose a possible link between the selective difficulty in recognising angry faces and STN stimulation affecting anterior cingulate cortex function.

Facial expressions of anger signal discontent with the behaviour of others and aim to discourage socially inappropriate and unexpected behaviour. ${ }^{22}$ An fMRI study has shown involvement of the orbitofrontal and medial prefrontal cortex in the processing of transgression of social norms. ${ }^{23}$ As both structures have previously been associated with the perception of angry faces, ${ }^{21}$ the authors suggested a possible role for social cues in modulating social behaviour. ${ }^{23}$

However, the relation between the perception of angry faces and social maladjustment in patients with Parkinson's disease receiving STN stimulation needs to be confirmed in future studies with combined investigation of facial recognition and neuropsychiatric inventory, as well as neurobehavioural measurements. 


\section{ACKNOWLEDGEMENTS}

This study was supported by the Deutsche Parkinson VereinigungBundesverband-e.V.

\section{Authors' affiliations}

U Schroeder, A Kuehler, A Hennenlotter, B Haslinger, A O CeballosBaumann, Department of Neurology, Technische Universität München, Munich, Germany

V M Tronnier, Department of Neurosurgery, Universität Heidelberg, Heidelberg, Germany

M Krause, Department of Neurology, Universität Heidelberg

R Pfister, Department of Neurology, Zentralklinikum Augsburg, Augsburg, Germany

R Sprengelmeyer, School of Psychology, University of St Andrews, St

Andrews, Fife, Scotland

K W Lange, Institute of Experimental Psychology, Universität

Regensburg, Regensburg, Germany

Competing interests: none declared

Correspondence to: Dr Andrés Ceballos-Baumann, Department of Neurology, Klinikum rechts der Isar, Technische Universität München, Möhlstr 28, D-81675 Munich, Germany; a.ceballos@|rz.tumuenchen.de

Received 3 June 2003

In revised form 23 July 2003

Accepted 23 August 2003

\section{REFERENCES}

1 Ceballos-Baumann AO, Boecker $\mathrm{H}$, Bartenstein $\mathrm{P}$, et al. A positron emission tomographic study of subthalamic nucleus stimulation in Parkinson disease: enhanced movement-related activity of motor-association cortex and decreased motor cortex resting activity. Arch Neurol 1999;56:997-1003

2 Limousin P, Greene J, Pollak P, et al. Changes in cerebral activity pattern due to subthalamic nucleus or internal pallidum stimulation in Parkinson's disease. Ann Neurol 1997:42:283-91.

3 Dujardin K, Defebvre L, Krystkowiak P, et al. Influence of chronic bilateral stimulation of the subthalamic nucleus on cognitive function in Parkinson's disease. J Neurol 2001;248:603-11.
4 Jahanshahi M, Ardouin C, Brown RG, et al. The impact of deep brain stimulation on executive function in Parkinson's disease. Brain 2000; 123:1 1 142-54.

5 Saint-Cyr JA, Trepanier LL, Kumar R, et al. Neuropsychological consequences of chronic bilateral stimulation of the subthalamic nucleus in Parkinson's disease. Brain 2000;123:2091-108

6 Schroeder U, Kuehler A, Haslinger B, et al. Subthalamic nucleus stimulation affects striato-anterior cingulate cortex circuit in a response conflict task: a PET study. Brain 2002;125:1995-2004.

7 Kulisevsky J, Berthier ML, Gironell A, et al. Mania following deep brain stimulation for Parkinson's disease. Neurology 2002;59:1421-4.

8 Hoveto JL, Mesnage V, Mallet L, et al. Behavioural disorders, Parkinson's disease and subthalamic stimulation. J Neurol Neurosurg Psychiatry 2002;72:701-7.

9 Ekman P. An argument for basic emotions. Cogn Emot 1992;6:169-200.

10 Folstein MF, Folstein SE, McHugh PR. "Mini-mental state". A practical method for grading the cognitive state of patients for the clinician. J Psychiatr Res 1975; 12:189-98.

11 Lehrl S. Manual zum MWT-B. Erlangen: Straube, 1977.

12 Wechsler D. Wechsler memory scale - revised. San Antonio: The Psychological Corporation, 1987.

13 Warrington EK, James M. VOSP: the visual object and space perception battery. Burry St Edmunds: Thames Valley Test Company, 1991.

14 Benton AL, Sivan AB, Hamsher KS, et al. Facial recognition. Stimulus and multiple choice pictures. New York: Oxford University Press, 1983.

15 Fahn S, Elton R, for the Unified Parkinson's Disease Rating Scale Development Committee. Unified Parkinson's disease rating scale, In: Fahn S, Marsden CD Goldstein M, eds. Recent developments in Parkinson's Disease. New York: Macmillan, 1987:153-63.

16 Hoehn MM, Yahr MD. Parkinsonism: onset, progression and mortality. Neurology 1967; 17:427-42.

17 Young AW, Perrett D, Calder A, et al. Facial expressions of emotions: stimuli and test (FEEST). Thurstone: Thames Valley Test Company, 2002.

18 Sprengelmeyer R, Young AW, Mahn K, et al. Facial expression recognition in people with medicated and unmedicated Parkinson's disease. Neuropsychologia 2003:41:1047-57.

19 Parent A, Hazrati LN. Functional anatomy of the basal ganglia II. The place of subthalamic nucleus and external pallidum in basal ganglia circuitry. Brain Res Brain Res Rev 1995;20:128-54.

20 Canteras NS, Shammah-Lagnado SJ, Silva BA, et al. Afferent connections of the subthalamic nucleus: a combined retrograde and anterograde horseradish peroxidase study in the rat. Brain Res 1990;513:43-59.

21 Blair RJR, Morris JS, Frith CD, et al. Dissociable neural responses to facial expressions of sadness and anger. Brain 1999;122:883-93.

22 Averill JR. Anger and aggression: an essay on emotion. New York: Springer, 1982.

23 Berthoz S, Armony JL, Blair RJ, et al. An FMRI study of intentional and unintentional (embarrassing) violations of social norms. Brain 2002;125:1696-708. 